\title{
ANALYSIS OF MODE I CONDUCTING CRACK IN PIEZO-ELECTRO-MAGNETO-ELASTIC LAYER
}

\author{
B. ROGOWSKI \\ Department of Mechanics of Materials \\ Technical University of Lodz \\ A1. Politechniki 6, 93-590 Łodz, POLAND \\ E-mail: bogdan.rogowski@p.lodz.pl
}

\begin{abstract}
Within the theory of linear magnetoelectroelasticity, the fracture analysis of a magneto - electrically dielectric crack embedded in a magnetoelectroelastic layer is investigated. The prescribed displacement, electric potential and magnetic potential boundary conditions on the layer surfaces are adopted. Applying the Hankel transform technique, the boundary - value problem is reduced to solving three coupling Fredholm integral equations of second kind. These equations are solved exactly. The corresponding semi - permeable crack - face magnetoelectric boundary conditions are adopted and the electric displacement and magnetic induction of crack interior are obtained explicitly. This field inside the crack is dependent on the material properties, applied loadings, the dielectric permittivity and magnetic permeability of crack interior, and the ratio of the crack length and the layer thickness. Field intensity factors are obtained as explicit expressions.
\end{abstract}

Key words: magneto electro elastic layer, Penny -shaped crack, dielectric crack, field intensity factors, exact solution.

\section{Introduction}

Materials having magnetoelectroelastic coupling effects have found increasing applications in engineering structures, particularly in smart materials intelligent structures. The effects of magnetoelectromechanical coupling have been observed in single phase materials where simultaneous magnetic and electric ordering coexists and in two phase composites where the participating phases are piezoelectric and piezomagnetic. These "smart" materials are extensively used as electric packaging, sensors and actuators, magnetic field probes, acoustic and ultrasonic devices, hydrophones and transducers with the responsibility of electromagnetomechanical energy conversion. When subjected to mechanical, magnetic and electrical loads in service, these magnetoelectroelastic composites can fail prematurely due to some defects, namely cracks, holes and others, arising during their manufacturing processes. Therefore, it is of great importance to study the magnetoelectroelastic interaction and fracture behaviours of magnetoelectroelastic materials. On the other hand, composites consisting of piezoelectric and piezomagnetic components have found their ways increasingly in applications in engineering structures. This is because these composites have some new properties of magnetoelectricity with the secondary piezoelectric effects which are not found in single phase piezoelectric or piezomagnetic materials. In some cases, the magnetoelectric effect of piezoelectric / piezomagnetic composites can be obtained by a hundred times longer than that of a single phase magnetoelectric material. Recently, Chen et al. (2004) derived a general solution for a transversely isotropic electromagnetothermoelastic material. In consequence, the components of the coupled field are expressed by five mono harmonic functions. More recently, a penny shaped crack in a magnetoelectroelastic material has been considered. For example, Zhao et al. (2006) analyzed a penny shaped crack in a magnetoelectroelastic medium. Niraula and Wang (2006) derived an exact closed form solution for a penny shaped crack in a magnetoelectrothermoelastic material in a temperature field. The electro magnetic field inside the crack was taken into account and closed form solutions were derived for an impermeable and permeable crack (Rogowski, 2011). Wang and Mai (2007) and Rogowski (2007) discussed the different 
electromagnetic boundary conditions on the crack- faces in PEMO - elastic materials. On the other hand, Zhong and Li (2007; 2008), Rogowski (2007), Zhong (2009) extended the semi permeable crack face electric boundary conditions proposed by Hao and Shen (1994) to analyze the PEMO elastic fields induced by dielectric cracks. However, all of the studies considered only infinite body and numerical procedures were used to obtained the results of approximate type. To the best of the author's knowledge, the penny shaped crack problems for the layer and limited permeable cracks have not been addressed yet, in an exact form. Motivated by this the author of this paper investigates a PEMO elastic layer, with an electrically and magnetically conducting crack under prescribed displacement, electric potential and magnetic potential boundary loading, to show exact solutions. Such solutions depend on a large number of material parameters, in our analysis it is seventeen, making any solution other than explicit analytical ones impractical.

\section{Basic equations in magnetoelectroelastic theory}

The constitutive equations within the framework of the linearly magnetoelectroelastic theory, in an axially symmetric problem, can be written as

$$
\begin{aligned}
& {\left[\begin{array}{c}
\sigma_{r} \\
\sigma_{\theta} \\
\sigma_{z} \\
D_{z} \\
B_{z}
\end{array}\right]=\left[\begin{array}{lllll}
c_{11} & c_{12} & c_{13} & e_{31} & q_{31} \\
c_{12} & c_{11} & c_{13} & e_{31} & q_{31} \\
c_{13} & c_{13} & c_{33} & e_{33} & q_{33} \\
e_{31} & e_{31} & e_{33} & -\varepsilon_{33} & -d_{33} \\
q_{31} & q_{31} & q_{33} & -d_{33} & -\mu_{33}
\end{array}\right]\left[\begin{array}{c}
u_{r, r} \\
u_{r} / r \\
u_{z, z} \\
\varphi_{, z} \\
\psi_{, z}
\end{array}\right],} \\
& {\left[\begin{array}{c}
\sigma_{r z} \\
D_{r} \\
B_{r}
\end{array}\right]=\left[\begin{array}{llll}
c_{44} & c_{44} & e_{15} & q_{15} \\
e_{15} & e_{15} & -\varepsilon_{11} & -d_{11} \\
q_{15} & q_{15} & -d_{11} & -\mu_{11}
\end{array}\right]\left[\begin{array}{c}
u_{r, z} \\
u_{z, r} \\
\varphi_{, r} \\
\psi_{, r}
\end{array}\right] \quad \psi_{, r}=\frac{\partial \psi}{\partial r},} \\
& E_{r}=-\varphi_{, r}, \quad E_{z}=-\varphi_{, z}, \quad H_{r}=-\psi_{, r}, \quad H_{z}=-\psi_{, z}
\end{aligned}
$$

where $u_{r}, u_{z}, \varphi, \psi$ are the elastic displacement, electric potential and magnetic potential, respectively; $\sigma_{r}$ , $\sigma_{\theta}, \sigma_{z}, \sigma_{r z}, D_{r}, D_{z}, B_{r}, B_{z}, E_{r}, E_{z}, H_{r}, H_{z}$ are the components of stress, electric displacement, magnetic induction, electric field and magnetic field, respectively; $e_{k l}, q_{k l}$ and $d_{k l}$ are the piezoelectric, piezomagnetic and magnetoelectric constants, respectively; $c_{k l}, \varepsilon_{k l}$ and $\mu_{k l}$ are the elastic stiffness, the dielectric permittivities and the magnetic permeabilities, respectively.

Moreover, from the equations of equilibrium

$$
\begin{array}{ll}
\sigma_{r, r}+\sigma_{r z, z}+\left(\sigma_{r}-\sigma_{\theta}\right) / r=0, & \\
\sigma_{r z, r}+\sigma_{z, z}+\left(\sigma_{r z}\right) / r=0, & \\
D_{r, r}+D_{z, z}+\left(D_{r}\right) / r=0, & \\
B_{r, r}+B_{z, z}+\left(B_{r}\right) / r=0, & B_{r, r}=\frac{\partial B_{r}}{\partial r},
\end{array}
$$

the elastic displacements, electric potential and magnetic potential will satisfy the basic governing equations as follows 


$$
\begin{aligned}
& c_{11} B_{1} u_{r}+c_{44} D^{2} u_{r}+\left(c_{13}+c_{44}\right) D \frac{\partial u_{z}}{\partial r}+\left(e_{31}+e_{15}\right) D \frac{\partial \varphi}{\partial r}+\left(q_{31}+q_{15}\right) D \frac{\partial \psi}{\partial r}=0, \\
& c_{44} B_{0} u_{z}+c_{33} D^{2} u_{z}+\left(c_{13}+c_{44}\right) D \frac{\partial\left[r u_{r}\right]}{r \partial r}+\left(e_{15} B_{0}+e_{33} D^{2}\right) \varphi+\left(q_{15} B_{0}+q_{33} D^{2}\right) \psi=0, \\
& \left(e_{31}+e_{15}\right) D \frac{\partial\left[r u_{r}\right]}{r \partial r}+\left(e_{15} B_{0}+e_{33} D^{2}\right) u_{z}-\left(\varepsilon_{11} B_{0}+\varepsilon_{33} D^{2}\right) \varphi-\left(d_{11} B_{0}+d_{33} D^{2}\right) \psi=0, \\
& \left(q_{31}+q_{15}\right) D \frac{\partial\left[r u_{r}\right]}{r \partial r}+\left(q_{15} B_{0}+q_{33} D^{2}\right) u_{z}-\left(d_{11} B_{0}+d_{33} D^{2}\right) \varphi-\left(\mu_{11} B_{0}+\mu_{33} D^{2}\right) \psi=0
\end{aligned}
$$

where the following differential operators are introduced

$$
B_{k}=\frac{\partial^{2}}{\partial r^{2}}+\frac{1}{r} \frac{\partial}{\partial r}-\frac{k}{r^{2}} ; \quad k=0,1 ; \quad D=\frac{\partial}{\partial z} ; \quad D^{2}=\frac{\partial^{2}}{\partial z^{2}}
$$

The general solution of Eqs (2.3) are as follows

$$
\begin{aligned}
& u_{r}(r, z)=\sum_{i=1}^{4} a_{1 i} \lambda_{i} \frac{\partial \varphi_{i}\left(r, z_{i}\right)}{\partial r} \\
& u_{z}(r, z)=\sum_{i=1}^{4} \frac{a_{2 i}}{\lambda_{i}} \frac{\partial \varphi_{i}\left(r, z_{i}\right)}{\partial z} \\
& \varphi(r, z)=-\sum_{i=1}^{4} \frac{a_{3 i}}{\lambda_{i}} \frac{\partial \varphi_{i}\left(r, z_{i}\right)}{\partial z} \\
& \psi(r, z)=-\sum_{i=1}^{4} \frac{a_{4 i}}{\lambda_{i}} \frac{\partial \varphi_{i}\left(r, z_{i}\right)}{\partial z}
\end{aligned}
$$

The functions $\varphi_{i}\left(r, z_{i}\right)(i=1,2,3,4)$ satisfy the following mono harmonic equations

$$
\left(\frac{\partial^{2}}{\partial r^{2}}+\frac{1}{r} \frac{\partial}{\partial r}+\frac{1}{\lambda_{i}^{2}} \frac{\partial^{2}}{\partial z^{2}}\right) \varphi_{i}\left(r, z_{i}\right)=0, \quad(i=1,2,3,4)
$$

where $z_{i}=\lambda_{i} z$ and $\lambda_{i}$, satisfying $\operatorname{Re}\left(\lambda_{i}\right)>0$, are the four eigenvalues of the characteristic equation which is an eight degree polynomial

$$
a \lambda^{8}+b \lambda^{6}+c \lambda^{4}+d \lambda^{2}+e=0
$$

The material parameters $a, b, c, d$ and $e$ are defined in the Appendix by Eq.(A1). The parameters $a_{j i} ; j=1,2,3,4 ; i=1,2,3,4$ are given as follows 


$$
a_{j i}=\frac{a_{j} \lambda_{i}^{6}+b_{j} \lambda_{i}^{4}+c_{j} \lambda_{i}^{2}+d_{j}}{a_{2} \lambda_{i}^{6}+b_{2} \lambda_{i}^{4}+c_{2} \lambda_{i}^{2}+d_{2}}
$$

where $a_{j}, b_{j}, c_{j}, d_{j}$ are given in the Appendix by Eq.(A2). Note that $a_{2 i} \equiv 1$.

Then from Eqs (2.1) together with (2.5) the components of stress, electric displacement and magnetic induction can be derived.

We have

$$
\begin{aligned}
& \sigma_{r}=-\sum_{i=1}^{4} \frac{a_{5 i}}{\lambda_{i}} \frac{\partial^{2} \varphi_{i}}{\partial z^{2}}-\left(c_{11}-c_{12}\right) \frac{u_{r}}{r}, \\
& \sigma_{\theta}=-\sum_{i=1}^{4} \frac{a_{5 i}}{\lambda_{i}} \frac{\partial^{2} \varphi_{i}}{\partial z^{2}}-\left(c_{11}-c_{12}\right) \frac{\partial u_{r}}{\partial r}, \\
& \sigma_{z}=\sum_{i=1}^{4} \frac{a_{5 i}}{\lambda_{i}^{3}} \frac{\partial^{2} \varphi_{i}}{\partial z^{2}}, \quad \sigma_{r z}=\sum_{i=1}^{4} \frac{a_{5 i}}{\lambda_{i}} \frac{\partial^{2} \varphi_{i}}{\partial r \partial z}, \\
& D_{r}=\sum_{i=1}^{4} a_{6 i} \lambda_{i} \frac{\partial^{2} \varphi_{i}}{\partial r \partial z}, \quad D_{z}=\sum_{i=1}^{4} \frac{a_{6 i}}{\lambda_{i}} \frac{\partial^{2} \varphi_{i}}{\partial z^{2}}, \\
& B_{r}=\sum_{i=1}^{4} a_{7 i} \lambda_{i} \frac{\partial^{2} \varphi_{i}}{\partial r \partial z}, \\
& B_{z}=\sum_{i=1}^{4} \frac{a_{7 i}}{\lambda_{i}} \frac{\partial^{2} \varphi_{i}}{\partial z^{2}}
\end{aligned}
$$

where

$$
\begin{aligned}
& a_{5 i}\left(q_{31}+q_{15}\right)=c_{44} q_{31}-c_{13} q_{15}+\left(c_{44} q_{31} \lambda_{i}^{2}+c_{11} q_{15}\right) a_{1 i}+\left(e_{31} q_{15}-e_{15} q_{31}\right) a_{3 i}, \\
& a_{6 i}\left(d_{11}-d_{33} \lambda_{i}^{2}\right)=d_{11} e_{33}-e_{15} d_{33}-\left(e_{15} d_{33} \lambda_{i}^{2}+e_{31} d_{11}\right) a_{1 i}+\left(\varepsilon_{33} d_{11}-\varepsilon_{11} d_{33}\right) a_{3 i}, \\
& a_{7 i}\left(\mu_{11}-\mu_{33} \lambda_{i}^{2}\right)=\mu_{11} q_{33}-q_{15} \mu_{33}-\left(q_{15} \mu_{33} \lambda_{i}^{2}+q_{31} \mu_{11}\right) a_{1 i}+\left(\mu_{11} d_{33}-\mu_{33} d_{11}\right) a_{3 i} .
\end{aligned}
$$

It should be noted that the general solutions given by Eqs (2.5) and (2.9) are valid for the cases when the eigenvalues $\lambda_{i}(i=1,2,3,4)$ are distinct. In this paper, equal roots (the special cases) are viewed as the limiting case of the distinct roots. For a pure piezoelectric medium we have $a_{7 i} \equiv 0$ and $a_{4 i} \equiv 0$ and

$$
\begin{aligned}
& a_{3 i}\left(e_{31}+e_{15}\right)=c_{13}+c_{44}-\left(c_{11}-c_{44} \lambda_{i}^{2}\right) a_{1 i}, \\
& a_{5 i}\left(e_{31}+e_{15}\right)=c_{44} e_{31}-c_{13} e_{15}+\left(c_{11} e_{15}+c_{44} e_{31} \lambda_{i}^{2}\right) a_{1 i}, \\
& a_{6 i}\left(\varepsilon_{11}-\varepsilon_{33} \lambda_{i}^{2}\right)=e_{33} \varepsilon_{11}-e_{15} \varepsilon_{33}-\left(e_{31} \varepsilon_{11}+e_{15} \varepsilon_{33} \lambda_{i}^{2}\right) a_{1 i} .
\end{aligned}
$$

It is easily verified, by direct substitution, that the equilibrium Eqs $(2.2)_{1,2}$, electric and magnetic charge conservation Eqs (2.2) 3,4 are satisfied by general solution (2.9). 


\section{Penny shaped dielectric and magnetic crack in the PEMO elastic layer}

Consider a penny shaped dielectric and assume that a magnetic crack is located in the middle plane of a transversely isotropic PEMO elastic layer as shown in Fig.1a. The cylindrical coordinate system $(r, \theta, z)$ is used with the poling axis as the $z$ axis. It is further assumed that the crack is centrally situated at the circle $r \leq a$ and the width of a layer is $2 h$. A constant displacement $\delta_{0}$, electric potential $\varphi_{0}$ and magnetic potential $\psi_{0}$ are imposed on the layer surfaces, namely

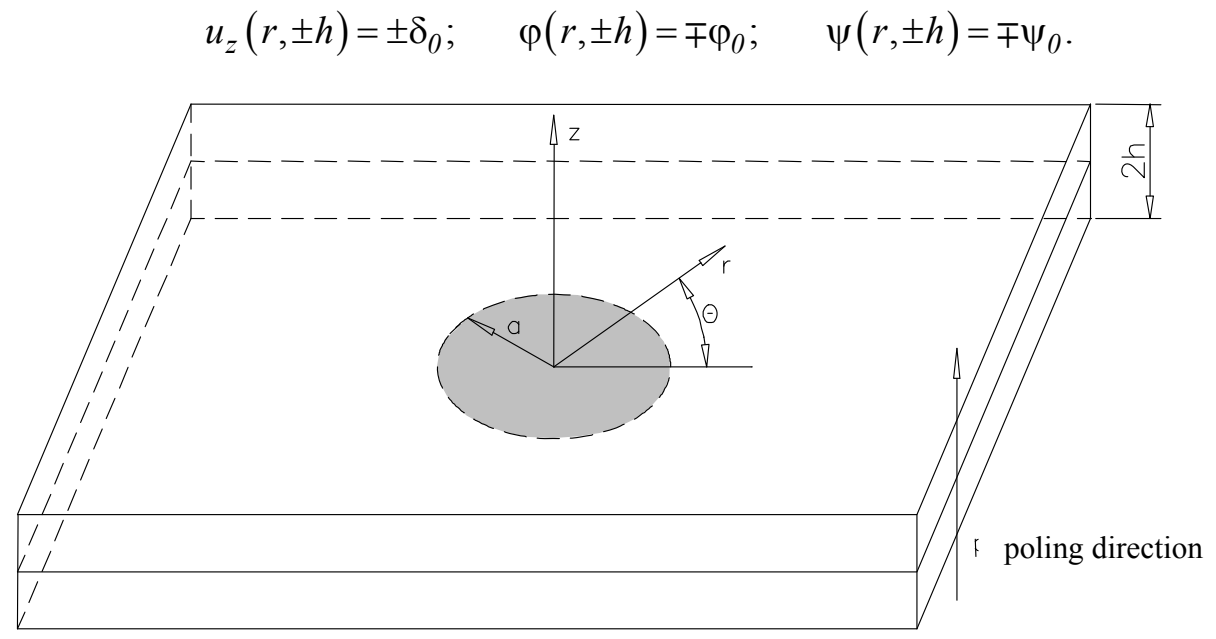

Fig.1a. Geometry of a magnetoelectroelastic layer with a penny shaped crack; the quantities $\sigma^{*}, D^{*}$ and $B^{*}$ are in the circular region $r \leq a$ in a plate without a crack.

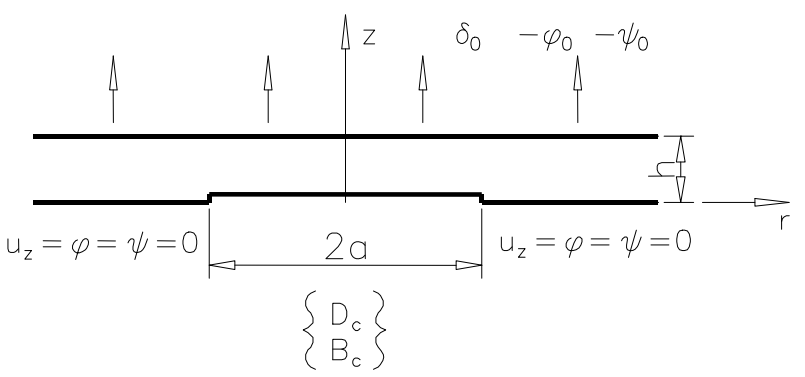

Fig.1b. The illustration of the boundary conditions; the following should be added to the crack surface: $-\sigma^{*}$, $-D^{*}$ and $-B^{*} ; \sigma_{z r}=0$ at $z=0$.

Equations (3.1) indicate that the top and bottom surfaces of the layer, $y= \pm h$, are sliding clamped and displaced along the $z$ direction by an amount of $2 \delta$ and there is a constant electric potential difference $-2 \varphi_{0}$ and a constant magnetic potential difference $-2 \psi_{0}$ between the top and bottom surfaces. These equations give, in the layer without the crack, the particular solution

$$
\begin{aligned}
& u_{z}(r, z)=\frac{\delta_{0} z}{h} ; \quad \varphi(r, z)=-\frac{\varphi_{0} z}{h} ; \quad \psi(r, z)=-\frac{\psi_{0} z}{h}, \\
& u_{r}(r, z)=-\frac{c_{13}}{c_{11}+c_{12}} \frac{\delta_{0} r}{h}=-v_{r z} \frac{\delta_{0} r}{h},
\end{aligned}
$$




$$
\begin{aligned}
& \sigma_{r}=\sigma_{\theta}=0 \\
& \sigma_{z}(r, z)=\left(c_{33}-\frac{2 c_{13}^{2}}{c_{11}+c_{12}}\right) \frac{\delta_{0}}{h}-e_{33} \frac{\varphi_{0}}{h}-q_{33} \frac{\psi_{0}}{h}=\tilde{E}_{z} \frac{\delta_{0}}{h}-e_{33} \frac{\varphi_{0}}{h}-q_{33} \frac{\psi_{0}}{h}, \\
& D_{z}=\left(e_{33}-\frac{2 c_{13}}{c_{11}+c_{12}} e_{31}\right) \frac{\delta_{0}}{h}+\varepsilon_{33} \frac{\varphi_{0}}{h}+d_{33} \frac{\psi_{0}}{h}=\left(e_{33}-v_{r z} e_{31}\right) \frac{\delta_{0}}{h}+\varepsilon_{33} \frac{\varphi_{0}}{h}+d_{33} \frac{\psi_{0}}{h}, \\
& B_{z}=\left(q_{33}-\frac{2 c_{13}}{c_{11}+c_{12}} q_{31}\right) \frac{\delta_{0}}{h}+d_{33} \frac{\varphi_{0}}{h}+\mu_{33} \frac{\psi_{0}}{h}=\left(q_{33}-v_{r z} q_{31}\right) \frac{\delta_{0}}{h}+d_{33} \frac{\varphi_{0}}{h}+\mu_{33} \frac{\psi_{0}}{h}, \\
& \sigma_{r z}=0 ; \quad E_{z}=\frac{\varphi_{0}}{h} ; \quad H_{z}=\frac{\psi_{0}}{h}
\end{aligned}
$$

where $\tilde{E}_{z}$ is the Young modulus in the $z$ direction.

By superposition principle the crack problem is equivalent to the perturbation problem under the applied loading on the crack surface and condition of symmetry on the plane $z=0$ outside the crack region.

$$
\begin{array}{ll}
\sigma_{z}(r, 0)=-\sigma^{*} ; & D_{z}(r, 0)=D_{c}-D^{*} ; \quad B_{z}(r, 0)=B_{c}-B^{*} ; \quad r<a, \\
\sigma_{z r}(r, 0)=0 ; & r \geq 0, \\
u_{z}(r, 0)=0 ; & \varphi(r, 0)=0 ; \quad \psi(r, 0)=0 ; \quad r \geq a
\end{array}
$$

where $\sigma^{*}, D^{*}, B^{*}$ are constant which from Eq.(3.2) are given by formulae

$$
\begin{aligned}
& \sigma^{*}=\tilde{E}_{z} \frac{\delta_{0}}{h}-e_{33} \frac{\varphi_{0}}{h}-q_{33} \frac{\psi_{0}}{h}, \\
& D^{*}=\left(e_{33}-v_{r z} e_{31}\right) \frac{\delta_{0}}{h}+\varepsilon_{33} \frac{\varphi_{0}}{h}+d_{33} \frac{\psi_{0}}{h}, \\
& B^{*}=\left(q_{33}-v_{r z} q_{31}\right) \frac{\delta_{0}}{h}+d_{33} \frac{\varphi_{0}}{h}+\mu_{33} \frac{\psi_{0}}{h} .
\end{aligned}
$$

In the above equations $\tilde{E}_{z}$ and $v_{r z}$ are the Young modulus and Poisson's ratio in the principal direction of anisotropy, the $z$ axis. In Eq.(3.3) $D_{c}$ and $B_{c}$ are normal components of the electric displacement and the magnetic induction, respectively, on the crack faces and inside the crack region, which for semi permeable crack face magnetoelectric boundary conditions are expressed as follows

$$
D_{c}=-\varepsilon_{c} \frac{\Delta \varphi}{\Delta u_{z}} ; \quad B_{c}=-\mu_{c} \frac{\Delta \psi}{\Delta u_{z}} ; \quad r \leq a
$$

where $\varepsilon_{c}=\varepsilon_{r} \varepsilon_{0} \quad\left(\varepsilon_{0}=8,85 \times 10^{-12} \mathrm{~F} / \mathrm{m}\right.$ the dielectric permittivity of air $)$ and $\mu_{c}=\mu_{r} \mu_{0}($ $\mu_{0}=4 \pi \times 10^{-7} N / A^{2}$ - the magnetic permeability of air) are the electric permittivity and magnetic 
permeability of the medium inside the crack; $\Delta \varphi, \Delta \psi$ and $\Delta u_{z}$ are the jumps of the electric potential, magnetic potential and crack opening displacement, respectively, across the crack. Especially, one can see that the crack reduces to an air when $\varepsilon_{r}=1$ and $\mu_{r}=1$. If the crack is filled by silicone oil, then $\varepsilon_{r}=2,5$; in the case of water $\varepsilon_{r}=81$.

\section{Solution method}

To solve the mixed boundary value problem on the crack plane, we express the solution for mono harmonic functions $\varphi_{i}\left(r, \lambda_{i} z\right)$ as the following Hankel integrals

$$
\varphi_{\mathrm{i}}\left(r, \lambda_{i} z\right)=\int_{0}^{\infty} \xi^{-1} A_{i}(\xi) \frac{\cosh \left[-\lambda_{i} \xi(z-h)\right]}{\sinh \left(\lambda_{i} \xi h\right)} J_{0}(\xi r) d \xi
$$

where $A_{i}(\xi)$ are the unknowns $(i=1,2,3,4)$ to be obtained from the boundary conditions and $J_{0}(\xi r)$ is the Bessel function of the first kind and zero order, and $\lambda_{i}$ are the roots of the characteristic Eq.(2.7). Since $\pm \lambda_{i}$ are the roots of the characteristic Eq.(2.7) it should be pointed out that the roots satisfying $\operatorname{Re}\left(\lambda_{j}\right)>0$ are only chosen and used in Eq (4.1) to satisfy the regularity condition at infinity.

Then from Eq.(2.9) the components of displacements $u_{r}$ and $u_{z}$, potentials $\varphi$ and $\psi$, stresses $\sigma_{r}$, $\sigma_{\theta}, \sigma_{z}, \sigma_{r z}$, electric displacement $D_{r}$ and $D_{z}$ and magnetic induction $B_{r}, B_{z}$ can be derived. We have

$$
\begin{aligned}
& u_{r}(r, z)=-\sum_{i=1}^{4} a_{1 i} \lambda_{i} \int_{0}^{\infty} A_{i}(\xi) \frac{\cosh \left[-\lambda_{i} \xi(z-h)\right]}{\sin \left(\lambda_{i} \xi h\right)} J_{l}(\xi r) d \xi-v_{r z} \frac{\delta_{0}}{h} r \\
& {\left[\begin{array}{c}
u_{z}(r, z) \\
\varphi(r, z) \\
\psi(r, z)
\end{array}\right]=\sum_{i=1}^{4}\left[\begin{array}{l}
-1 \\
a_{3 i} \\
a_{4 i}
\end{array}\right] \int_{0}^{\infty} A_{i}(\xi) \frac{\sinh \left[-\lambda_{i} \xi(z-h)\right]}{\sinh \left(\lambda_{i} \xi h\right)} J_{0}(\xi r) d \xi+\left[\begin{array}{c}
\frac{\delta_{0}}{h} z \\
-\frac{\varphi_{0}}{h} z \\
-\frac{\psi_{0}}{h} z
\end{array}\right],} \\
& {\left[\begin{array}{l}
\sigma_{r}(r, z) \\
\sigma_{\theta}(r, z) \\
\sigma_{z}(r, z) \\
D_{z}(r, z) \\
B_{z}(r, z)
\end{array}\right]=\sum_{i=1}^{4}\left[\begin{array}{l}
-a_{5 i} \lambda_{i} \\
a_{5 i} / \lambda_{i} \\
a_{6 i} \lambda_{i} \\
a_{7 i} \lambda_{i}
\end{array}\right] \int_{0}^{\infty} \xi A_{i}(\xi) \frac{\cosh \left[-\lambda_{i} \xi(z-h)\right]}{\sinh \left(\lambda_{i} \xi h\right)} J_{0}(\xi r) d \xi+} \\
& {\left[\begin{array}{c}
\left(c_{11}-c_{12}\right) \\
\left(c_{11}-c_{12}\right)\left(\frac{\partial u_{r}}{\partial r}+v_{r z} \frac{\delta_{0}}{h}\right) \\
0 \\
0 \\
0
\end{array}\right]+\left[\begin{array}{l}
0 \\
0 \\
\sigma_{r z}^{*} \\
\sigma^{*} \\
D^{*} \\
B^{*}
\end{array}\right],}
\end{aligned}
$$




$$
\left[\begin{array}{c}
\sigma_{r z}(r, z) \\
D_{r}(r, z) \\
B_{r}(r, z)
\end{array}\right]=\sum_{i=1}^{4}\left[\begin{array}{c}
a_{5 i} \\
a_{6 i} \lambda_{i}^{2} \\
a_{7 i} \lambda_{i}^{2}
\end{array}\right] \int_{0}^{\infty} \xi A_{i}(\xi) \frac{\sinh \left[-\lambda_{i} \xi(z-h)\right]}{\sinh \left(\lambda_{i} \xi h\right)} J_{l}(\xi r) d \xi
$$

The resulting expressions and boundary conditions (3.3), (3.4) and (3.5) are

$$
\begin{aligned}
& {\left[\begin{array}{c}
\sigma_{z}(r, 0) \\
D_{z}(r, 0) \\
B_{z}(r, 0)
\end{array}\right]=\sum_{i=1}^{4}\left[\begin{array}{c}
a_{5 i} / \lambda_{i} \\
a_{6 i} \lambda_{i} \\
a_{7 i} \lambda_{i}
\end{array}\right] \int_{0}^{\infty} \xi A_{i}(\xi) \operatorname{coth}\left(\lambda_{i} \xi h\right) J_{0}(\xi r) d \xi=\left[\begin{array}{c}
-\sigma^{*} \\
D_{c}-D^{*} \\
B_{c}-B^{*}
\end{array}\right] ; \quad r<a,} \\
& \sigma_{r z}(r, 0)=\sum_{i=1}^{4} a_{5 i} \int_{0}^{\infty} \xi A_{i}(\xi) J_{l}(\xi r) d \xi=0 ; \quad r \geq 0, \\
& {\left[\begin{array}{c}
u_{z}(r, 0) \\
\varphi(r, 0) \\
\psi(r, 0)
\end{array}\right]=\sum_{i=1}^{4}\left[\begin{array}{c}
-1 \\
a_{3 i} \\
a_{4 i}
\end{array}\right] \int_{0}^{\infty} A_{i}(\xi) J_{0}(\xi r) d \xi=0 ; \quad r \geq a .}
\end{aligned}
$$

On the plane $z=h$ we have satisfied the boundary conditions (3.1) and $\sigma_{r z}(r, h) \equiv 0$ and in addition

$$
\begin{aligned}
& u_{r}(r, h)=-\sum_{i=1}^{4} a_{1 i} \lambda_{i} \int_{0}^{\infty} A_{i}(\xi) \frac{1}{\sin \left(\lambda_{i} \xi h\right)} J_{1}(\xi r) d \xi-v_{r z} \frac{\delta_{0}}{h} r, \\
& \sigma_{z}(r, h)=\sum_{i=1}^{4} \frac{a_{5 i}}{\lambda_{i}} \int_{0}^{\infty} \xi A_{i}(\xi) \frac{1}{\sinh \left(\lambda_{i} \xi h\right)} J_{0}(\xi r) d \xi+\sigma^{*} .
\end{aligned}
$$

The boundary conditions (3.1), which give the boundary values (4.9), are of sliding clamped type with prescribed axial displacement $\delta_{0}$ and electric, and magnetic potentials $\varphi_{0}$ and $\psi_{0}$, respectively.

For convenience, we introduce three new functions $U(\xi), \Phi(\xi)$ and $\Psi(\xi)$, such that

$$
\sum_{i=1}^{4}\left[\begin{array}{c}
1 \\
a_{3 i} \\
a_{4 i} \\
a_{5 i}
\end{array}\right] A_{i}(\xi)=\left[\begin{array}{c}
U(\xi) \\
\Phi(\xi) \\
\Psi(\xi) \\
0
\end{array}\right] .
$$

Then the unknowns $A_{i}(\xi)$ can be written as

$$
\left[\begin{array}{c}
A_{1}(\xi) \\
A_{2}(\xi) \\
A_{3}(\xi) \\
A_{4}(\xi)
\end{array}\right]=\left[b_{j i}\right]_{4 \times 4}\left[\begin{array}{c}
U(\xi) \\
\Phi(\xi) \\
\Psi(\xi) \\
0
\end{array}\right]
$$

where 


$$
\left[b_{j i}\right]_{4 \times 4}=\left[\begin{array}{cccc}
1 & 1 & 1 & 1 \\
a_{31} & a_{32} & a_{33} & a_{34} \\
a_{41} & a_{42} & a_{43} & a_{44} \\
a_{51} & a_{52} & a_{53} & a_{54}
\end{array}\right]^{-1},
$$

and where " -1 " denotes the inverse matrix.

The constants $A_{i}(\xi)$ are obtained as follows

where

$$
m_{2} A_{i}(\xi)=d_{i} U(\xi)+l_{i} \Phi(\xi)+k_{i} \Psi(\xi)
$$

$$
m_{2}=\sum_{i=1}^{4} d_{i}=\sum_{i=1}^{4} a_{3 i} l_{i}=\sum_{i=1}^{4} a_{4 i} k_{i} .
$$

We have the identities

$$
\begin{aligned}
& \sum l_{i}=\sum k_{i}=\sum a_{3 i} d_{i}=\sum a_{3 i} k_{i}=\sum a_{4 i} d_{i}=\sum a_{4 i} l_{i}= \\
& =\sum a_{5 i} d_{i}=\sum a_{5 i} l_{i}=\sum a_{5 i} k_{i} \equiv 0
\end{aligned}
$$

where sums are from 1 to 4 .

The material parameters $d_{i}, l_{i}$, and $k_{i} ; i=1,2,3,4$, are

$$
\begin{aligned}
& d_{1}=a_{52}\left(a_{33} a_{44}-a_{43} a_{34}\right)+a_{53}\left(a_{34} a_{42}-a_{32} a_{44}\right)+a_{54}\left(a_{32} a_{43}-a_{33} a_{42}\right), \\
& d_{2}=a_{51}\left(a_{34} a_{43}-a_{33} a_{44}\right)+a_{53}\left(a_{31} a_{44}-a_{34} a_{41}\right)+a_{54}\left(a_{33} a_{41}-a_{31} a_{43}\right), \\
& d_{3}=a_{51}\left(a_{32} a_{44}-a_{34} a_{42}\right)+a_{52}\left(a_{34} a_{41}-a_{31} a_{44}\right)+a_{54}\left(a_{31} a_{42}-a_{32} a_{41}\right), \\
& d_{4}=a_{51}\left(a_{33} a_{42}-a_{32} a_{43}\right)+a_{52}\left(a_{31} a_{43}-a_{33} a_{41}\right)+a_{53}\left(a_{32} a_{41}-a_{31} a_{42}\right), \\
& l_{1}=a_{52}\left(a_{43}-a_{44}\right)+a_{53}\left(a_{44}-a_{42}\right)+a_{54}\left(a_{42}-a_{43}\right), \\
& l_{2}=a_{51}\left(a_{44}-a_{43}\right)+a_{53}\left(a_{41}-a_{44}\right)+a_{54}\left(a_{43}-a_{41}\right), \\
& l_{3}=a_{51}\left(a_{42}-a_{44}\right)+a_{52}\left(a_{44}-a_{41}\right)+a_{54}\left(a_{41}-a_{42}\right), \\
& l_{4}=a_{51}\left(a_{43}-a_{42}\right)+a_{52}\left(a_{41}-a_{43}\right)+a_{53}\left(a_{42}-a_{41}\right), \\
& k_{1}=a_{52}\left(a_{34}-a_{33}\right)+a_{53}\left(a_{32}-a_{34}\right)+a_{54}\left(a_{33}-a_{32}\right), \\
& k_{2}=a_{51}\left(a_{33}-a_{34}\right)+a_{53}\left(a_{34}-a_{31}\right)+a_{54}\left(a_{31}-a_{33}\right), \\
& k_{3}=a_{51}\left(a_{34}-a_{32}\right)+a_{52}\left(a_{31}-a_{34}\right)+a_{54}\left(a_{32}-a_{31}\right), \\
& k_{4}=a_{51}\left(a_{32}-a_{33}\right)+a_{52}\left(a_{33}-a_{31}\right)+a_{53}\left(a_{31}-a_{32}\right) .
\end{aligned}
$$


The boundary conditions (4.6), (4.7) and (4.8) give a system of coupled integral equations for $U(\xi)$, $\Phi(\xi)$ and $\Psi(\xi)$ in the following form

$$
\begin{aligned}
& m_{2} \sigma_{z}(r, 0)=\int_{0}^{\infty}\left[m U(\xi)+m_{6} \Phi(\xi)+\tilde{m}_{6} \Psi(\xi)\right] \xi J_{0}(\xi r) d \xi+ \\
& +\sum_{1}^{4} \frac{a_{5 i}}{\lambda_{i}} \int_{0}^{\infty}\left[d_{i} U(\xi)+l_{i} \Phi(\xi)+k_{i} \Psi(\xi)\right]\left[\operatorname{coth}\left(\lambda_{i} \xi h\right)-1\right] \xi J_{0}(\xi r) d \xi=-m_{2} \sigma^{*} ; \quad r<a, \\
& m_{2} D_{z}(r, 0)=\int_{0}^{\infty}\left[m_{5} U(\xi)+m_{7} \Phi(\xi)+m_{8} \Psi(\xi)\right] \xi J_{0}(\xi r) d \xi+ \\
& +\sum_{i=1}^{4} a_{6 i} \lambda_{i} \int_{0}^{\infty}\left[d_{i} U(\xi)+l_{i} \Phi(\xi)+k_{i} \Psi(\xi)\right]\left[\operatorname{coth}\left(\lambda_{i} \xi h\right)-1\right] \xi J_{0}(\xi r) d \xi=m_{2}\left(D_{c}-D^{*}\right) ; \quad r<a \\
& m_{2} B_{z}(r, 0)=\int_{0}^{\infty}\left[m_{9} U(\xi)+m_{10} \Phi(\xi)+m_{11} \Psi(\xi)\right] \xi J_{0}(\xi r) d \xi+ \\
& +\sum_{i=1}^{4} a_{7 i} \lambda_{i} \int_{0}^{\infty}\left[d_{i} U(\xi)+l_{i} \Phi(\xi)+k_{i} \Psi(\xi)\right]\left[\operatorname{coth}\left(\lambda_{i} \xi h\right)-1\right] \xi J_{0}(\xi r) d \xi=m_{2}\left(B_{c}-B^{*}\right) ; \quad r<a \\
& {\left[\begin{array}{l}
u_{z}(r, 0) \\
\varphi(r, 0) \\
\psi(r, 0)
\end{array}\right]=\int_{0}^{\infty}\left[\begin{array}{l}
-U(\xi) \\
(\xi(\xi) \\
\Psi(\xi)
\end{array}\right] J_{0}(\xi r) d \xi=0 ; \quad r \geq a,}
\end{aligned}
$$

where

$$
\begin{array}{lll}
m=\sum_{1}^{4} \frac{a_{5 i}}{\lambda_{i}} d_{i} ; & m_{6}=\sum_{1}^{4} \frac{a_{5 i}}{\lambda_{i}} l_{i} ; & \tilde{m}_{6}=\sum_{1}^{4} \frac{a_{5 i}}{\lambda_{i}} k_{i}, \\
m_{5}=\sum_{1}^{4} a_{6 i} \lambda_{i} d_{i} ; & m_{7}=\sum_{1}^{4} a_{6 i} \lambda_{i} l_{i} ; & m_{8}=\sum_{1}^{4} a_{6 i} \lambda_{i} k_{i}, \\
m_{9}=\sum_{1}^{4} a_{7 i} \lambda_{i} d_{i} ; & m_{10}=\sum_{1}^{4} a_{7 i} \lambda_{i} l_{i} ; & m_{11}=\sum_{1}^{4} a_{7 i} \lambda_{i} k_{i} .
\end{array}
$$

Introduce the new functions $f_{i}(x)(i=1,2,3)$ and the following Fourier integral representation of the functions $U(\xi), \Phi(\xi)$ and $\Psi(\xi)$

$$
\left[\begin{array}{l}
U(\xi) \\
\Phi(\xi) \\
\Psi(\xi)
\end{array}\right]=\int_{0}^{\infty}\left[\begin{array}{l}
f_{1}(s) \\
f_{2}(s) \\
f_{3}(s)
\end{array}\right] \sin (\xi s) d s ; \quad f_{i}(0)=0
$$


then

$$
\left[\begin{array}{c}
u_{z}(r, 0) \\
\varphi(r, 0) \\
\psi(r, 0)
\end{array}\right]=\int_{0}^{a}\left[\begin{array}{c}
-f_{l}(s) \\
f_{2}(s) \\
f_{3}(s)
\end{array}\right] d s \int_{0}^{\infty} \sin (\xi s) J_{0}(\xi r) d \xi
$$

Recalling the following knows result

$$
\int_{0}^{\infty} \sin (\xi s) J_{0}(\xi r) d \xi=\frac{H(s-r)}{\sqrt{s^{2}-r^{2}}}
$$

where $H($.$) is the Heaviside function, we find that the boundary conditions (4.20) are automatically$ satisfied. Moreover, the displacement, electric potential and magnetic potential on the crack plane can be expressed in terms of the introduced unknown functions as

$$
\left[\begin{array}{c}
u_{z}(r, 0) \\
\varphi(r, 0) \\
\psi(r, 0)
\end{array}\right]=\int_{r}^{a}\left[\begin{array}{c}
-f_{l}(s) \\
f_{2}(s) \\
f_{3}(s)
\end{array}\right] \frac{d s}{\sqrt{s^{2}-r^{2}}}
$$

Multiplying both sides of Eqs (4.17), (4.18) and (4.19) by $r / \sqrt{s^{2}-r^{2}}$, integrating with respect to $r$ from 0 to $x \quad(x<0)$, respectively, and using the following identities

$$
\int_{0}^{x} \frac{r J_{0}(\xi r)}{\sqrt{x^{2}-r^{2}}} d r=\frac{\sin (\xi x)}{\xi}, \quad \int_{0}^{\infty} \sin (\xi s) \sin (\xi x) d \xi=\frac{\pi}{2} \delta(s-x)
$$

where $\delta(\cdot)$ is the Dirac delta function, Eqs (4.17), (4.18) and (4.19) can be rewritten as

$$
\begin{aligned}
& m f_{1}(x)-m_{6} f_{2}(x)-\tilde{m}_{6} f_{3}(x)+ \\
& +\frac{2}{\pi} \sum_{1}^{4} \frac{a_{5 i}}{\lambda_{i}} \int_{0}^{a}\left[d_{i} f_{1}(s)-l_{i} f_{2}(s)-k_{i} f_{3}(s)\right] K_{i}(x, s) d s=\frac{2}{\pi} \sigma^{*} m_{2} x, \\
& m_{5} f_{l}(x)-m_{7} f_{2}(x)-m_{8} f_{3}(x)+ \\
& +\frac{2}{\pi} \sum_{1}^{4} a_{6 i} \lambda_{i} \int_{0}^{a}\left[d_{i} f_{1}(s)-l_{i} f_{2}(s)-k_{i} f_{3}(s)\right] K_{i}(x, s) d s=\frac{2}{\pi}\left(D^{*}-D_{c}\right) m_{2} x \\
& m_{9} f_{1}(x)-m_{10} f_{2}(x)-m_{11} f_{3}(x)+ \\
& +\frac{2}{\pi} \sum_{1}^{4} a_{7 i} \lambda_{i} \int_{0}^{a}\left[d_{i} f_{l}(s)-l_{i} f_{2}(s)-k_{i} f_{3}(s)\right] K_{i}(x, s) d s=\frac{2}{\pi}\left(B^{*}-B_{c}\right) m_{2} x
\end{aligned}
$$

where the kernel functions $K_{i}(x, s)$ are defined as follows 


$$
K_{i}(x, s)=\frac{2}{\pi} \int_{0}^{\infty}\left[\operatorname{coth}\left(\lambda_{i} \xi h\right)-1\right] \sin (\xi s) \sin (\xi x) d \xi, \quad i=1,2,3,4 .
$$

Next, the solutions of electric displacement and magnetic induction inside the crack are of interest. Application of Eqs (3.7) and (4.25) leads to

$$
\begin{array}{ll}
D_{c} \int_{r}^{a} \frac{f_{1}(s)}{\sqrt{s^{2}-r^{2}}} d s-\varepsilon_{r} \varepsilon_{0} \int_{r}^{a} \frac{f_{2}(s)}{\sqrt{s^{2}-r^{2}}} d s=0 ; & 0 \leq r \leq a, \\
B_{c} \int_{r}^{a} \frac{f_{1}(s)}{\sqrt{s^{2}-r^{2}}} d s-\mu_{r} \mu_{0} \int_{r}^{a} \frac{f_{3}(s)}{\sqrt{s^{2}-r^{2}}} d s=0 ; & 0 \leq r \leq a .
\end{array}
$$

Differentiating both Eqs (4.29) with respect to $r$ and using the following rule of differentiation under integral sign

$$
\frac{d}{d r} \int_{r}^{a} \frac{f(s)}{\sqrt{s^{2}-r^{2}}} d s=-\frac{r f(a)}{a \sqrt{a^{2}-r^{2}}}+r \int_{r}^{a} \frac{d}{d s}\left(\frac{f(s)}{s}\right) \frac{d s}{\sqrt{s^{2}-r^{2}}} .
$$

Equations (4.29) may be rewritten as follows

$$
\begin{aligned}
& -\frac{r}{a \sqrt{a^{2}-r^{2}}}\left[D_{c} f_{l}(a)-\varepsilon_{r} \varepsilon_{0} f_{2}(a)\right]+ \\
& +r\left[D_{c} \int_{r}^{a} \frac{d}{d s}\left(\frac{f_{1}(s)}{s}\right) \frac{d s}{\sqrt{s^{2}-r^{2}}}-\varepsilon_{r} \varepsilon_{0} \int_{r}^{a} \frac{d}{d s}\left(\frac{f_{2}(s)}{s}\right) \frac{d s}{\sqrt{s^{2}-r^{2}}}\right]=0, \\
& -\frac{r}{a \sqrt{a^{2}-r^{2}}}\left[B_{c} f_{1}(a)-\mu_{r} \mu_{0} f_{3}(a)\right]+ \\
& +r\left[B_{c} \int_{r}^{a} \frac{d}{d s}\left(\frac{f_{l}(s)}{s}\right) \frac{d s}{\sqrt{s^{2}-r^{2}}}-\mu_{r} \mu_{0} \int_{r}^{a} \frac{d}{d s}\left(\frac{f_{3}(s)}{s}\right) \frac{d s}{\sqrt{s^{2}-r^{2}}}\right]=0 ; \quad 0 \leq r \leq a .
\end{aligned}
$$

The first terms in both Eqs (4.31) are singular at $r \rightarrow a-0$, while other terms tend to zero in at this point. For the singularity to vanish at $r \rightarrow a-0$, it must be true that

$$
\begin{aligned}
& D_{c} f_{1}(a)-\varepsilon_{r} \varepsilon_{0} f_{2}(a)=0, \\
& B_{c} f_{1}(a)-\mu_{r} \mu_{0} f_{3}(a)=0 .
\end{aligned}
$$

Equations (4.32) determine unknown quantities $D_{c}$ and $B_{c}$. The relations are non linear since $f_{l}(a)$, $f_{2}(a)$ and $f_{3}(a)$ depend also on $D_{c}$ and $B_{c}$, as shown in Eqs (4.27). Equations (4.32) form two coupling quadratic equations with respect to $D_{c}$ and $B_{c}$. Those are dependent on the material properties, electric permittivity and magnetic permeability of the crack interior and applied loadings. In addition, it is found that 
the kernel function $K_{i}(x, s ; h)$ depends on the width of the layer $D_{c}$ and $B_{c}$ also depends on $h$. Moreover, although these are at most four pairs of roots of $D_{c}$ and $B_{c}$ according to the nonlinear Eqs (4.32) only one pair is reasonable and the other are superfluous. The acceptable $D_{c}$ and $B_{c}$ should be located at the range between that for a magneto electrically impermeable crack (zeroes values) and that for a magneto - electrically permeable crack (the extremally possible values). Four ideal crack face electromagnetic boundary conditions: (i) $D_{c}=0$ and $B_{c}=0$, (ii) $D_{c} \neq 0$ and $B_{c}=0$, (iii) $D_{c}=0$ and $B_{c} \neq 0$, (iv) $D_{c} \neq 0$ and $B_{c} \neq 0$ are the limiting cases of the electromagnetically semi permeable crack model ("dielectric and magnetic crack").

\section{The exact solution of Fredholm integral equation}

Using the known result

$$
\operatorname{coth}\left(\lambda_{i} \xi h\right)-1=2 \sum_{n=1}^{\infty} e^{-2 n \lambda_{i} \xi h}
$$

we find that the kernel function (4.28) is

$$
K_{i}(x, s)=\sum_{n=1}^{\infty}\left(\frac{2 n \lambda_{i} h}{\left(2 n \lambda_{i} h\right)^{2}+(x-s)^{2}}-\frac{2 n \lambda_{i} h}{\left(2 n \lambda_{i} h\right)^{2}+(x+s)^{2}}\right) .
$$

Consequently, Eqs (4.27) can be rewritten as

$$
\begin{aligned}
& \Lambda\left[\begin{array}{l}
f_{1}(x) \\
f_{2}(x) \\
f_{3}(x)
\end{array}\right]+ \\
& +\frac{2}{\pi} \sum_{i=1}^{4} \sum_{n=1}^{\infty}\left[\begin{array}{c}
a_{5 i} / \lambda_{i} \\
a_{6 i} \lambda_{i} \\
a_{7 i} \lambda_{i}
\end{array}\right] \int_{0}^{a}\left[d_{i} f_{l}(s)-l_{i} f_{2}(s)-k_{i} f_{3}(s)\right]\left[\frac{2 n \lambda_{i} h}{\left(2 n \lambda_{i} h\right)^{2}+(x-s)^{2}}-\frac{2 n \lambda_{i} h}{\left(2 n \lambda_{i} h\right)^{2}+(x+s)^{2}}\right] d s=(5.3) \\
& =\frac{2}{\pi} m_{2} x\left[\begin{array}{c}
\sigma^{*} \\
D^{*}-D_{c} \\
B^{*}-B_{c}
\end{array}\right] .
\end{aligned}
$$

This Fredholm integral equation of the second kind (5.3) can be solved explicitly. The method of consecutive iteration yields the $N$ th approximation

$$
\begin{aligned}
& {\left[\begin{array}{l}
f_{1 N}(x) \\
f_{2 N}(x) \\
f_{3 N}(x)
\end{array}\right]=\left[\begin{array}{l}
f_{10}(x) \\
f_{20}(x) \\
f_{30}(x)
\end{array}\right]+} \\
& -\sum_{i=1}^{4} \sum_{n=1}^{\infty}\left[\begin{array}{c}
a_{5 i} / \lambda_{i} \\
a_{6 i} \lambda_{i} \\
a_{7 i} \lambda_{i}
\end{array}\right]\left[d_{i} f_{10}(x)-l_{i} f_{20}(x)-k_{i} f_{30}(x)\right]\left[F_{i n}(x)-F_{i n}^{2}(x)+F_{i n}^{3}(x)-\ldots+F_{i n}^{N}(x)\right]
\end{aligned}
$$


where

$$
\begin{aligned}
& {\left[\begin{array}{l}
f_{10}(x) \\
f_{20}(x) \\
f_{30}(x)
\end{array}\right]=\frac{2}{\pi} m_{2} x \Lambda^{-1}\left[\begin{array}{c}
\sigma^{*} \\
D^{*}-D_{c} \\
B^{*}-B_{c}
\end{array}\right],} \\
& F_{i n}(x)=\frac{2}{\pi}\left(\frac{n \lambda_{i} h}{x} \ln \left(\frac{\left(2 n \lambda_{i} h\right)^{2}+(x-a)^{2}}{\left(2 n \lambda_{i} h\right)^{2}+(x+a)^{2}}\right)+\tan ^{-1}\left(\frac{x+a}{2 n \lambda_{i} h}\right)-\tan ^{-1}\left(\frac{x-a}{2 n \lambda_{i} h}\right)\right), \\
& \Lambda=\left[\begin{array}{ccc}
m & -m_{6} & -\tilde{m}_{6} \\
m_{5} & -m_{7} & -m_{8} \\
m_{9} & -m_{10} & -m_{11}
\end{array}\right],
\end{aligned}
$$

and the superscript " -1 " denotes the inverse of a matrix $\Lambda$.

The sum of infinite geometric series converges to the solution as $N \rightarrow \infty$, giving

$$
\begin{aligned}
& {\left[\begin{array}{l}
f_{1}(x) \\
f_{2}(x) \\
f_{3}(x)
\end{array}\right]=\frac{2}{\pi} m_{2} x \Lambda^{-1}\left[\begin{array}{c}
\sigma^{*} \\
D^{*}-D_{c} \\
B^{*}-B_{c}
\end{array}\right]+} \\
& -\sum_{i=1}^{4} \sum_{n=1}^{\infty}\left[\begin{array}{c}
a_{5 i} / \lambda_{i} \\
a_{6 i} \lambda_{i} \\
a_{7 i} \lambda_{i}
\end{array}\right]\left[d_{i} f_{10}(x)-l_{i} f_{20}(x)-k_{i} f_{30}(x)\right] \frac{F_{i n}(x)}{1+F_{i n}(x)}
\end{aligned}
$$

The range of convergence is given by the inequality

$$
\left|F_{\text {in }}(x)\right|<1,
$$

and is satisfied for all of $0 \leq x \leq a$ and $a / h$. For the limiting case of an infinite magnetoelectroelastic space $F_{i n}(x, a / h) \rightarrow 0$ as $h \rightarrow \infty$. On the other hand, for a very thin plate $F_{i n}(x, a / h)$ tends to unity since $h \rightarrow 0$. To check the above results, it is natural to consider the special case where a dielectric crack is embedded in an infinite magnetoelectroelastic material, i.e., $h \rightarrow \infty$. One can find that the solution may be solved explicitly. This solution is given in Appendix $B$.

\section{Analysis of field intensity factors}

Defining the field intensity factors as follows

$$
K_{q}=\lim _{r \rightarrow a^{+}} \sqrt{2 \pi(r-a)} q(r)
$$

where $q$ stands for $\sigma_{z}, D_{z}$ and $B_{z}$, respectively, we then find that the intensity factors of stress, electric displacement and magnetic induction can be expressed as 


$$
\begin{aligned}
& {\left[\begin{array}{c}
K_{\sigma} \\
K_{D} \\
K_{B}
\end{array}\right]=\frac{2}{\pi} \sqrt{\pi a}\left\{\left[\begin{array}{c}
\sigma^{*} \\
D^{*}-D_{c} \\
B^{*}-B_{c}
\end{array}\right]+\right.} \\
& \left.-\frac{\Lambda}{m_{2}} \sum_{i=1}^{4} \sum_{n=1}^{\infty}\left[\begin{array}{c}
a_{5 i} / \lambda_{i} \\
a_{6 i} \lambda_{i} \\
a_{7 i} \lambda_{i}
\end{array}\right]\left[d_{i} f_{10}(a)-l_{i} f_{20}(a)-k_{i} f_{30}(a)\right] \frac{2}{\pi} \frac{\tan ^{-1}\left(\alpha_{i n}\right)-\frac{1}{\alpha_{i n}} \ln \left(1+\alpha_{i n}^{2}\right)}{1+\frac{2}{\pi}\left(\tan ^{-1}\left(\alpha_{i n}\right)-\frac{1}{\alpha_{i n}} \ln \left(1+\alpha_{i n}^{2}\right)\right)}\right\}
\end{aligned}
$$

where

$$
\alpha_{i n}=\frac{a}{\lambda_{i} n h} .
$$

The first closed form solutions, for an infinite medium, are identical with the known result given by Zhong and Li (2007) through a different approach. Similarly, the field intensity factors associated with the crack opening displacement $u_{z}(r)$, electric potential $\varphi(r)$ and magnetic potential $\psi(r)$ across the crack near the crack front are defined and easily derived from Eqs (4.25) as

$$
\begin{aligned}
& K_{C O D} \stackrel{\Delta}{=} \lim _{r \rightarrow a^{-}} \sqrt{\frac{\pi}{2(a-r)}} u_{z}(r)=\sqrt{\pi a} \frac{f_{1}(a)}{a}, \\
& K_{\varphi} \stackrel{\Delta}{=} \lim _{r \rightarrow a^{-}} \sqrt{\frac{\pi}{2(a-r)}} \varphi(r)=\sqrt{\pi a} \frac{f_{2}(a)}{a}, \\
& K_{\Psi} \stackrel{\Delta}{=} \lim _{r \rightarrow a^{-}} \sqrt{\frac{\pi}{2(a-r)}} \psi(r)=\sqrt{\pi a} \frac{f_{3}(a)}{a} .
\end{aligned}
$$

Figures 2 and 3 show the variation of the functions $f(\alpha)$ and $f(\alpha) /(1+f(\alpha))$, respectively.

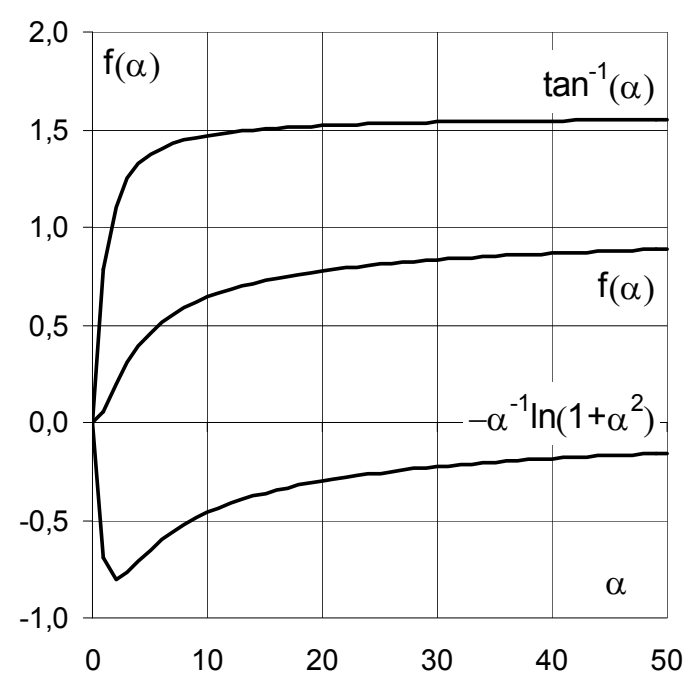

Fig.2. The variation of the function $f(\alpha)=\frac{2}{\pi}\left[\tan ^{-1}(\alpha)-\alpha^{-1} \ln \left(1+\alpha^{2}\right)\right]$ with $\alpha$. 


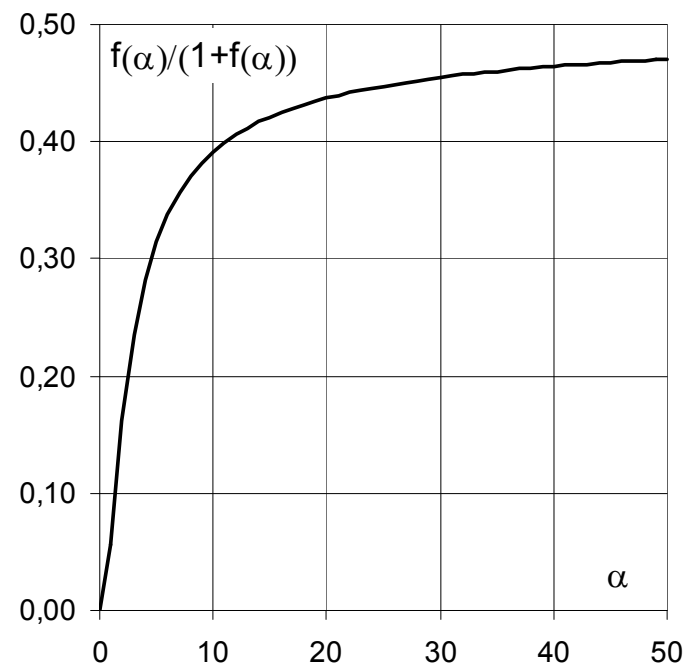

Fig.3. The variation of the function $f(\alpha) /(1+f(\alpha))$ with $\alpha$.

These field intensity factors can be presented explicitly

$$
\begin{aligned}
& {\left[\begin{array}{c}
K_{C O D} \\
K_{\varphi} \\
K_{\psi}
\end{array}\right]=\frac{2}{\pi} \sqrt{\pi a} m_{2} \Lambda^{-1}\left[\begin{array}{c}
\sigma^{*} \\
D^{*}-D_{c} \\
B^{*}-B_{c}
\end{array}\right]+} \\
& \left.-\sum_{i=1}^{4} \sum_{n=1}^{\infty}\left[\begin{array}{c}
a_{5 i} / \lambda_{i} \\
a_{6 i} \lambda_{i} \\
a_{7 i} \lambda_{i}
\end{array}\right]\left[d_{i} f_{10}(a)-l_{i} f_{20}(a)-k_{i} f_{30}(a)\right] \frac{2}{\pi} \frac{\tan ^{-1}\left(\alpha_{i n}\right)-\frac{1}{\alpha_{i n}} \ln \left(1+\alpha_{i n}^{2}\right)}{1+\frac{2}{\pi}\left(\tan ^{-1}\left(\alpha_{i n}\right)-\frac{1}{\alpha_{i n}} \ln \left(1+\alpha_{i n}^{2}\right)\right)}\right\} .
\end{aligned}
$$

Note that

$$
\left[\begin{array}{c}
K_{\sigma} \\
K_{D} \\
K_{B}
\end{array}\right]=\frac{\Lambda}{m_{2}}\left[\begin{array}{c}
K_{C O D} \\
K_{\varphi} \\
K_{\psi}
\end{array}\right]
$$

where the matrix $\Lambda / m_{2}$ may be partitioned as

$$
\left[\begin{array}{ccc}
\text { elastic stiffness } & \text { piezoelectric } & \text { piezomagnetic } \\
\text { piezoelectric } & \text { dielectric } & \text { magnetoelectric } \\
\text { piezomagnetic } & \text { magnetoelectric } & \text { magnetic }
\end{array}\right] \text {. }
$$

For the piezoelectric barium titanate $\mathrm{BaTiO}_{3}$ and piezomagnetic cobalt iron oxide $\mathrm{CoFe}_{2} \mathrm{O}_{4}$ composite (roughly 50:50 percent) we have

$$
\left[\begin{array}{ccc}
62,5 \times 10^{9} \mathrm{~N} / \mathrm{m}^{2} & 14,3 \mathrm{C} / \mathrm{m}^{2} & 7,0 \times 10^{3} \mathrm{~N} / \mathrm{Am} \\
14,3 \mathrm{C} / \mathrm{m}^{2} & -17,0 \times 10^{-9} \mathrm{C} / \mathrm{Vm} & -16,7 \times 10^{-9} \mathrm{C} / \mathrm{Am} \\
7,0 \times 10^{3} \mathrm{~N} / \mathrm{Am} & -16,7 \times 10^{-9} \mathrm{C} / \mathrm{Am} & -8,2 \times 10^{-6} \mathrm{~N} / \mathrm{A}^{2}
\end{array}\right] .
$$


The non zero magnetoelectric constant $d_{11}^{*}=-16,7 \times 10^{-9} \mathrm{C} / \mathrm{Am}$ exists only in the piezoelectric / piezomagnetic composite as a significant new feature.

The electric displacement $D_{c}$ and magnetic induction $B_{c}$ inside the crack are obtained from Eqs (4.32), i.e.,

$$
\begin{aligned}
& D_{c}=\varepsilon_{r} \varepsilon_{0} f_{2}(a) / f_{1}(a), \\
& B_{c}=\mu_{r} \mu_{0} f_{3}(a) / f_{l}(a)
\end{aligned}
$$

where

$$
\begin{aligned}
& {\left[\begin{array}{l}
f_{1}(a) \\
f_{2}(a) \\
f_{3}(a)
\end{array}\right]=\frac{2}{\pi} m_{2} a \Lambda^{-1}\left[\begin{array}{c}
\sigma^{*} \\
D^{*}-D_{c} \\
B^{*}-B_{c}
\end{array}\right]+} \\
& -\sum_{i=1}^{4} \sum_{n=1}^{\infty}\left[\begin{array}{c}
a_{5 i} / \lambda_{i} \\
a_{6 i} \lambda_{i} \\
a_{7 i} \lambda_{i}
\end{array}\right]\left[d_{i} f_{10}(a)-l_{i} f_{20}(a)-k_{i} f_{30}(a)\right] \frac{2}{\pi} \frac{\tan ^{-1}\left(\alpha_{i n}\right)-\frac{1}{\alpha_{i n}} \ln \left(1+\alpha_{i n}^{2}\right)}{1+\frac{2}{\pi}\left(\tan ^{-1}\left(\alpha_{i n}\right)-\frac{1}{\alpha_{i n}} \ln \left(1+\alpha_{i n}^{2}\right)\right)}
\end{aligned}
$$

\section{Magnetoelectrically permeable crack}

For a magnetoelectrically permeable crack case both electric and magnetic potentials are continuous across the crack surfaces. Thus the problem can be reduced to the following Fredholm integral equation of the second kind

$$
\begin{aligned}
& m f_{1}(x)+\frac{2}{\pi} \sum_{i=1}^{4} \frac{a_{5 i}}{\lambda_{i}} d_{i} \int_{0}^{a} f_{l}(s) K_{i}\left(x, s, \lambda_{i} h\right) d s=\frac{2}{\pi} \sigma^{*} m_{2} x ; \quad x<a \\
& \text { and } \quad f_{2}(x)=0 ; \quad \text { and } \quad f_{3}(x)=0 .
\end{aligned}
$$

The solution of the integral equation is given explicitly

$$
f_{1}(x)=\frac{2}{\pi} \sigma^{*} x \frac{m_{2}}{m}\left[1-\sum_{i=1}^{4} \sum_{n=1}^{\infty} \frac{a_{5 i}}{\lambda_{i}} d_{i} \frac{F_{i n}(x)}{1+F_{\text {in }}(x)}\right] .
$$

The field intensity factors can finally be expressed as

$$
\begin{aligned}
& K_{C O D}=\frac{2}{\pi} \sigma^{*} \frac{m_{2}}{m} \sqrt{\pi a}\left[1-\sum_{i=1}^{4} \sum_{n=1}^{\infty} \frac{a_{5 i}}{\lambda_{i}} d_{i} \frac{\frac{2}{\pi}\left(\tan ^{-1}\left(\alpha_{i n}\right)-\frac{1}{\alpha_{i n}} \ln \left(1+\alpha_{i n}^{2}\right)\right)}{1+\frac{2}{\pi}\left(\tan ^{-1}\left(\alpha_{i n}\right)-\frac{1}{\alpha_{i n}} \ln \left(1+\alpha_{i n}^{2}\right)\right)}\right], \\
& K_{\sigma}=\frac{m}{m_{2}} K_{C O D} ; \quad K_{D}=\frac{m_{5}}{m_{2}} K_{C O D} ; \quad K_{B}=\frac{m_{9}}{m_{2}} K_{C O D} ; \quad K_{\varphi}=0 ; \quad K_{\psi}=0 .
\end{aligned}
$$


Equations (7.3) indicate that the four field intensity factors of COD, stress, electric displacement and magnetic induction depend on one another through material constants and thickness of the layer. In addition, $D_{c}$ and $B_{c}$ have no effect on these field intensity factors. For the barium titanate cobalt iron oxide composite material the elastic stiffness $m / m_{2}$ piezoelectric coefficient $m_{5} / m_{2}$ and piezomagnetic coefficient $m_{9} / m_{2}$ are obtained as follows: $62,5 \times 10^{9} \mathrm{~N} / \mathrm{m}^{2} ; 14,3 \mathrm{C} / \mathrm{m}^{2} ; 7,0 \times 10^{3} \mathrm{~N} / \mathrm{Am}$.

\section{Appendix A}

The material parameters in the characteristic Eq.(2.7) are as follows

$$
\begin{aligned}
& a=c_{44}\left[\mu_{33} e_{33}^{2}+\varepsilon_{33} q_{33}^{2}+c_{33} \mu_{33} \varepsilon_{33}-d_{33}\left(c_{33} d_{33}+2 e_{33} q_{33}\right)\right], \\
& b=\mu_{33}\left\{\left(e_{31}+e_{15}\right)\left[2 c_{13} e_{33}-c_{33}\left(e_{31}+e_{15}\right)\right]+2 c_{44} e_{33} e_{31}-c_{11} e_{33}^{2}-c_{33} c_{44} \varepsilon_{11}\right\}+ \\
& +\varepsilon_{33}\left\{\left(q_{31}+q_{15}\right)\left[2 c_{13} q_{33}-c_{33}\left(q_{31}+q_{15}\right)\right]+2 c_{44} q_{33} q_{31}-c_{11} q_{33}^{2}-c_{33} c_{44} \mu_{11}\right\}+ \\
& -\mu_{33} \varepsilon_{33} \tilde{c}^{2}-\left(e_{31}+e_{15}\right)^{2} q_{33}^{2}-\left(q_{31}+q_{15}\right)^{2} e_{33}^{2}-c_{44} \mu_{11} e_{33}^{2}-c_{44} \varepsilon_{11} q_{33}^{2}+ \\
& +2 e_{33} q_{33}\left(q_{31}+q_{15}\right)\left(e_{31}+e_{15}\right)+d_{33}^{2} \tilde{c}^{2}+2 c_{33} d_{33}\left(e_{31}+e_{15}\right)\left(q_{31}+q_{15}\right)+ \\
& +2 c_{44} c_{33} d_{11} d_{33}+2 e_{33} q_{33}\left(c_{44} d_{11}+c_{11} d_{33}\right)-2 d_{33}\left(c_{13}+c_{44}\right)\left[e_{33}\left(q_{31}+q_{15}\right)+q_{33}\left(e_{31}+e_{15}\right)\right] \text {, } \\
& c=\mu_{33}\left\{2 e_{15}\left[c_{11} e_{33}-c_{13}\left(e_{31}+e_{15}\right)\right]+c_{44} e_{31}^{2}+\varepsilon_{11} \tilde{c}^{2}\right\}+ \\
& +\varepsilon_{33}\left\{2 q_{15}\left[c_{11} q_{33}-c_{13}\left(q_{31}+q_{15}\right)\right]+c_{44} q_{31}^{2}+\mu_{11} \tilde{c}^{2}\right\}+ \\
& +c_{33} c_{44} \mu_{11} \varepsilon_{11}+c_{11} c_{44} \mu_{33} \varepsilon_{33}+2\left(c_{13}+c_{44}\right)\left(q_{31}+q_{15}\right)\left(d_{11} e_{33}+d_{33} e_{15}-q_{33} \varepsilon_{11}\right)+ \\
& +2\left(c_{13}+c_{44}\right)\left(e_{31}+e_{15}\right)\left(d_{11} q_{33}+d_{33} q_{15}-e_{33} \mu_{11}\right)+ \\
& +\left(q_{31}+q_{15}\right)^{2}\left(c_{33} \varepsilon_{11}+2 e_{33} e_{15}\right)+\left(e_{31}+e_{15}\right)^{2}\left(c_{33} \mu_{11}+2 q_{33} q_{15}\right)+ \\
& -2\left(q_{31}+q_{15}\right)\left(e_{31}+e_{15}\right)\left(e_{33} q_{15}+q_{33} e_{15}+c_{33} d_{11}+c_{44} d_{33}\right)+ \\
& -2 c_{11} d_{33}\left(e_{33} q_{15}+q_{33} e_{15}\right)-2 c_{44} d_{11}\left(q_{33} e_{15}+e_{33} q_{15}\right)+ \\
& -2 c_{11} d_{11} q_{33} e_{33}-2 c_{44} d_{33} q_{15} e_{15}+2 c_{44} q_{15} q_{33} \varepsilon_{11}+2 c_{44} e_{15} e_{33} \mu_{11}+ \\
& +c_{11} q_{33}^{2} \varepsilon_{11}+c_{11} e_{33}^{2} \mu_{11}-2 \tilde{c}^{2} d_{33} d_{11}-c_{11} c_{44} d_{33}^{2}-c_{44} c_{33} d_{11}^{2} \\
& d=-c_{11} \mu_{33}\left(c_{44} \varepsilon_{11}+e_{15}^{2}\right)-c_{11} \varepsilon_{33}\left(c_{44} \mu_{11}+q_{15}^{2}\right)-c_{44}\left(e_{31}^{2} \mu_{11}+q_{31}^{2} \varepsilon_{11}\right)+ \\
& -e_{31}^{2} q_{15}^{2}-q_{31}^{2} e_{15}^{2}-\mu_{11} \varepsilon_{11} \tilde{c}^{2}+d_{11} \tilde{c}^{2}+2 c_{11} c_{44} d_{11} d_{33}+ \\
& +2 c_{13} q_{15} q_{31} \varepsilon_{11}+2 c_{13} e_{15} e_{31} \mu_{11}-2 c_{11} q_{15} q_{33} \varepsilon_{11}-2 c_{11} e_{15} e_{33} \mu_{11}+ \\
& +2 c_{13} q_{15}^{2} \varepsilon_{11}+2 c_{13} e_{15}^{2} \mu_{11}+2 e_{31} e_{15} q_{31} q_{15}+2 c_{11} e_{15} q_{15} d_{33}+ \\
& +d_{11}\left[-2 c_{13} e_{15}\left(q_{15}+q_{31}\right)-2 c_{13} q_{15}\left(e_{15}+e_{31}\right)\right]+d_{11}\left[2 c_{11}\left(e_{15} q_{33}+q_{15} e_{33}\right)+2 c_{44} e_{31} q_{31}\right] \text {, } \\
& e=c_{11}\left[\mu_{11} e_{15}^{2}+\varepsilon_{11} q_{15}^{2}+c_{44} \varepsilon_{11} \mu_{11}-d_{11}\left(c_{44} d_{11}+2 e_{15} q_{15}\right)\right], \\
& \tilde{c}^{2}=c_{11} c_{33}-c_{13}\left(c_{13}+2 c_{44}\right) \text {. }
\end{aligned}
$$


Material coefficients in Eqs (2.8)

$$
\begin{aligned}
& a_{1}=\left(c_{13}+c_{44}\right)\left(\varepsilon_{33} q_{33}-e_{33} d_{33}\right)-\left(q_{31}+q_{15}\right)\left(c_{33} \varepsilon_{33}+e_{33}^{2}\right)+\left(e_{31}+e_{15}\right)\left(e_{33} q_{33}+c_{33} d_{33}\right) \text {, } \\
& b_{1}=\left(c_{13}+c_{44}\right)\left(e_{33} d_{11}+e_{15} d_{33}-\varepsilon_{33} q_{15}-\varepsilon_{11} q_{33}\right)+\left(q_{31}+q_{15}\right)\left(c_{44} \varepsilon_{33}+c_{33} \varepsilon_{11}+2 e_{33} e_{15}\right)+ \\
& -\left(e_{31}+e_{15}\right)\left(c_{44} d_{33}+c_{33} d_{11}+q_{33} e_{15}+e_{33} q_{15}\right) \text {, } \\
& c_{1}=\left(c_{13}+c_{44}\right)\left(\varepsilon_{11} q_{15}-e_{15} d_{11}\right)-\left(q_{31}+q_{15}\right)\left(c_{44} \varepsilon_{11}+e_{15}^{2}\right)+\left(e_{31}+e_{15}\right)\left(c_{44} d_{11}+e_{15} q_{15}\right), \\
& d_{1}=0, \quad a_{2}=c_{44}\left(e_{33} d_{33}-\varepsilon_{33} q_{33}\right), \\
& b_{2}=c_{11}\left(q_{33} \varepsilon_{33}-d_{33} e_{33}\right)+c_{44}\left(q_{15} \varepsilon_{33}+q_{33} \varepsilon_{11}-e_{15} d_{33}-d_{11} e_{33}\right)+ \\
& +\left(c_{13}+c_{44}\right)\left[d_{33}\left(e_{31}+e_{15}\right)-\varepsilon_{33}\left(q_{31}+q_{15}\right)\right]-\left(e_{31}+e_{15}\right)\left[e_{33}\left(q_{31}+q_{15}\right)-q_{33}\left(e_{31}+e_{15}\right)\right], \\
& c_{2}=c_{44}\left(d_{11} e_{15}-\varepsilon_{11} q_{15}\right)+\left(c_{13}+c_{44}\right)\left[\varepsilon_{11}\left(q_{31}+q_{15}\right)-d_{11}\left(e_{31}+e_{15}\right)\right]+ \\
& +\left(e_{31}+e_{15}\right)\left[e_{15}\left(q_{31}+q_{15}\right)-q_{15}\left(e_{31}+e_{15}\right)\right]+c_{11}\left(d_{11} e_{33}+d_{33} e_{15}-q_{15} \varepsilon_{33}-q_{33} \varepsilon_{11}\right), \\
& d_{2}=c_{11}\left(q_{15} \varepsilon_{11}-e_{15} d_{11}\right), \quad a_{3}=c_{44}\left(c_{33} d_{33}+e_{33} q_{33}\right), \\
& b_{3}=-c_{11}\left(c_{33} d_{33}+e_{33} q_{33}\right)-c_{44}\left(c_{44} d_{33}+e_{15} q_{33}+c_{33} d_{11}+q_{15} e_{33}\right)+ \\
& +\left(c_{13}+c_{44}\right)\left[\left(c_{13}+c_{44}\right) d_{33}+\left(q_{31}+q_{15}\right) e_{33}\right]+\left(e_{31}+e_{15}\right)\left[\left(c_{13}+c_{44}\right) q_{33}-\left(q_{31}+q_{15}\right) c_{33}\right] \text {, } \\
& c_{3}=c_{11}\left(c_{44} d_{33}+c_{33} d_{11}+e_{15} q_{33}+q_{15} e_{33}\right)+c_{44}\left(c_{44} d_{11}+q_{15} e_{15}\right)+ \\
& -\left(c_{13}+c_{44}\right)\left[\left(c_{13}+c_{44}\right) d_{11}+\left(q_{31}+q_{15}\right) e_{15}\right]-\left(e_{31}+e_{15}\right)\left[\left(c_{13}+c_{44}\right) q_{15}-\left(q_{31}+q_{15}\right) c_{44}\right] \text {, } \\
& d_{3}=-c_{11}\left(c_{44} d_{11}+e_{15} q_{15}\right), \quad a_{4}=-c_{44}\left(c_{33} \varepsilon_{33}+e_{33}^{2}\right) \\
& b_{4}=c_{11}\left(c_{33} \varepsilon_{33}+e_{33}^{2}\right)+c_{44}\left(c_{44} \varepsilon_{33}+c_{33} \varepsilon_{11}+2 e_{15} e_{33}\right)+ \\
& -\left(c_{13}+c_{44}\right)\left[\left(c_{13}+c_{44}\right) \varepsilon_{33}+\left(e_{31}+e_{15}\right) e_{33}\right]-\left(e_{31}+e_{15}\right)\left[\left(c_{13}+c_{44}\right) e_{33}-\left(e_{31}+e_{15}\right) c_{33}\right] \text {, } \\
& c_{4}=-c_{11}\left(c_{44} \varepsilon_{33}+c_{33} \varepsilon_{11}+2 e_{15} e_{33}\right)-c_{44}\left(c_{44} \varepsilon_{11}+e_{15}^{2}\right) \\
& +\left(c_{13}+c_{44}\right)\left[\left(c_{13}+c_{44}\right) \varepsilon_{11}+\left(e_{31}+e_{15}\right) e_{15}\right]+\left(e_{31}+e_{15}\right)\left[\left(c_{13}+c_{44}\right) e_{15}-\left(e_{31}+e_{15}\right) c_{44}\right] \text {, } \\
& d_{4}=c_{11}\left(c_{44} \varepsilon_{11}+e_{15}^{2}\right)
\end{aligned}
$$

The coefficients $a_{4}, b_{4}, c_{4}, d_{4}$ are obtained from coefficients $a_{3}, b_{3}, c_{3}, d_{3}$ by replacement of $d_{i i}$ by $\varepsilon_{i i}$ and $q_{k l}$ by $e_{k l}$ and changing the signum. 
If only a pure piezoelectric or pure piezomagnetic material is considered, then the electric or magnetic potential is defined by a polynomial of $\lambda$ which is given as follows $c_{44} c_{33} \lambda^{4}-\left[c_{11} c_{33}-c_{13}\left(c_{13}+2 c_{44}\right)\right] \lambda^{2}+c_{11} c_{44}$. This polynominal vanishes for a pure elastic transversely isotropic material without piezoelectromagnetic properties.

The roots of the characteristic Eq.(2.7) are presented by formulae

$$
\begin{aligned}
& \lambda_{1}^{2}=-\frac{b}{4 a}-\frac{1}{2} \sqrt{R_{5}+R_{6}}-\frac{1}{2} \sqrt{2 R_{5}-R_{6}+\frac{1}{4} \frac{R_{7}}{\sqrt{R_{5}+R_{6}}}}, \\
& \lambda_{2}^{2}=-\frac{b}{4 a}-\frac{1}{2} \sqrt{R_{5}+R_{6}}+\frac{1}{2} \sqrt{2 R_{5}-R_{6}+\frac{1}{4} \frac{R_{7}}{\sqrt{R_{5}+R_{6}}}}, \\
& \lambda_{3}^{2}=-\frac{b}{4 a}+\frac{1}{2} \sqrt{R_{5}+R_{6}}-\frac{1}{2} \sqrt{2 R_{5}-R_{6}-\frac{1}{4} \frac{R_{7}}{\sqrt{R_{5}+R_{6}}}}, \\
& \lambda_{4}^{2}=-\frac{b}{4 a}+\frac{1}{2} \sqrt{R_{5}+R_{6}}+\frac{1}{2} \sqrt{2 R_{5}-R_{6}-\frac{1}{4} \frac{R_{7}}{\sqrt{R_{5}+R_{6}}}}
\end{aligned}
$$

where

$$
\begin{array}{ll}
R_{1}=2 c^{3}-9 b c d+27 a d^{2}+27 b^{2} e-72 a c e ; & R_{2}=c^{2}-3 b d+12 a e, \\
R_{3}=\sqrt{R_{1}^{2}-4 R_{2}^{3}} ; \quad R_{4}=\sqrt[3]{\frac{1}{2}\left(R_{1}+R_{3}\right)}, & \\
R_{5}=\frac{b^{2}}{4 a^{2}}-\frac{2 c}{3 a} ; \quad R_{6}=\frac{R_{2}}{3 a R_{4}}+\frac{R_{4}}{3 a} ; \quad R_{7}=\frac{b^{3}}{a^{3}}-\frac{4 b c}{a^{2}}+\frac{8 d}{a} .
\end{array}
$$

\section{Appendix B}

Denoting

$$
\begin{aligned}
& \tilde{d}_{i}=\left(m_{12} d_{i}-m_{15} l_{i}-m_{18} k_{i}\right) \sigma^{*}-\left(m_{13} d_{i}-m_{16} l_{i}-m_{19} k_{i}\right)\left(D^{*}-D_{c}\right)+ \\
& -\left(m_{14} d_{i}-m_{17} l_{i}-m_{20} k_{i}\right)\left(B^{*}-B_{c}\right), \\
& \tilde{m}=m_{12}-m_{5} m_{13}-m_{9} m_{14}=m_{12}-m_{6} m_{15}-\tilde{m}_{6} m_{18}=m_{7} m_{16}-m_{5} m_{13}+m_{8} m_{19}= \\
& =m_{10} m_{17}+m_{11} m_{20}-m_{9} m_{14}, \\
& m_{12}=m_{7} m_{11}-m_{8} m_{10}, \quad m_{13}=m_{6} m_{11}-\tilde{m}_{6} m_{10}, \quad m_{14}=\tilde{m}_{6} m_{7}-m_{6} m_{8}, \\
& m_{15}=m_{5} m_{11}-m_{8} m_{9}, \quad m_{16}=m_{11}-\tilde{m}_{6} m_{9}, \quad m_{17}=\tilde{m}_{6} m_{5}-m m_{8}, \\
& m_{18}=m_{9} m_{7}-m_{5} m_{10}, \quad m_{19}=m_{9} m_{6}-m m_{10}, \quad m_{20}=m m_{7}-m_{6} m_{5}
\end{aligned}
$$

the physical quantities are obtained as follows 


$$
\begin{aligned}
& u_{r}(r, z)=\frac{r}{\pi \tilde{m}} \sum_{i=1}^{4} a_{1 i} \lambda_{i} \tilde{d}_{i}\left(\frac{\pi}{2}-\tan ^{-1} \zeta_{i}-\frac{\zeta_{i}}{1+\zeta_{i}^{2}}\right)-v_{r z} \frac{\delta_{0}}{h} r \\
& u_{z}(r, z)=\frac{2 a}{\pi \tilde{m}} \sum_{i=1}^{4} \eta_{i} \tilde{d}_{i}\left(1-\zeta_{i}\left(\frac{\pi}{2}-\tan ^{-1} \zeta_{i}\right)\right)+\frac{\delta_{0}}{h} z \\
& \varphi(r, z)=-\frac{2 a}{\pi \tilde{m}} \sum_{i=1}^{4} a_{3 i} \eta_{i} \tilde{d}_{i}\left(1-\zeta_{i}\left(\frac{\pi}{2}-\tan ^{-1} \zeta_{i}\right)\right)-\frac{\varphi_{0}}{h} z, \\
& \psi(r, z)=-\frac{2 a}{\pi \tilde{m}} \sum_{i=1}^{4} a_{4 i} \eta_{i} \tilde{d}_{i}\left(1-\zeta_{i}\left(\frac{\pi}{2}-\tan ^{-1} \zeta_{i}\right)\right)-\frac{\psi_{0}}{h} z, \\
& \sigma_{z r}(r, z)=-\frac{2 r}{\pi a \tilde{m}} \sum_{i=1}^{4} a_{5 i} \tilde{d}_{i} \frac{\eta_{i}}{\left(1+\zeta_{i}^{2}\right)\left(\zeta_{i}^{2}+\eta_{i}^{2}\right)}, \\
& \sigma_{z z}(r, z)=-\frac{2}{\pi \tilde{m}} \sum_{i=1}^{4} \frac{a_{5 i}}{\lambda_{i}} \tilde{d}_{i}\left(\frac{\pi}{2}-\tan ^{-1} \zeta_{i}-\frac{\zeta_{i}}{\zeta_{i}^{2}+\eta_{i}^{2}}\right)+\sigma^{*}, \\
& \sigma_{r r}(r, z)=\frac{2}{\pi \tilde{m}} \sum_{i=1}^{4} a_{5 i} \lambda_{i} \tilde{d}_{i}\left(\frac{\pi}{2}-\tan ^{-1} \zeta_{i}-\frac{\zeta_{i}}{\zeta_{i}^{2}+\eta_{i}^{2}}\right)-\left(c_{11}-c_{12}\right)\left(\frac{u_{r}}{r}+v_{r z} \frac{\delta_{0}}{h}\right), \\
& \sigma_{\theta \theta}(r, z)=\frac{2}{\pi \tilde{m}} \sum_{i=1}^{4} a_{5 i} \lambda_{i} \tilde{d}_{i}\left(\frac{\pi}{2}-\tan ^{-1} \zeta_{i}-\frac{\zeta_{i}}{\zeta_{i}^{2}+\eta_{i}^{2}}\right)-\left(c_{11}-c_{12}\right)\left(\frac{\partial u_{r}}{\partial r}+v_{r z} \frac{\delta_{0}}{h}\right) \\
& E_{r}(r, z)=-\frac{2 r}{\pi a \tilde{m}} \sum_{i=1}^{4} a_{3 i} \tilde{d}_{i} \frac{\eta_{i}}{\left(1+\zeta_{i}^{2}\right)\left(\zeta_{i}^{2}+\eta_{i}^{2}\right)} \\
& E_{z}(r, z)=-\frac{2}{\pi \tilde{m}} \sum_{i=1}^{4} a_{3 i} \lambda_{i} \tilde{d}_{i}\left(\frac{\pi}{2}-\tan ^{-1} \zeta_{i}-\frac{\zeta_{i}}{\zeta_{i}^{2}+\eta_{i}^{2}}\right)+\frac{\varphi_{0}}{h}, \\
& H_{r}(r, z)=-\frac{2 r}{\pi a \tilde{m}} \sum_{i=1}^{4} a_{4 i} \tilde{d}_{i} \frac{\eta_{i}}{\left(1+\zeta_{i}^{2}\right)\left(\zeta_{i}^{2}+\eta_{i}^{2}\right)}, \\
& H_{z}(r, z)=-\frac{2}{\pi \tilde{m}} \sum_{i=1}^{4} a_{4 i} \lambda_{i} \tilde{d}_{i}\left(\frac{\pi}{2}-\tan ^{-1} \zeta_{i}-\frac{\zeta_{i}}{\zeta_{i}^{2}+\eta_{i}^{2}}\right)+\frac{\psi_{0}}{h},
\end{aligned}
$$




$$
\begin{aligned}
& D_{r}(r, z)=-\frac{2 r}{\pi a \tilde{m}} \sum_{i=1}^{4} a_{6 i} \lambda_{i}^{2} \tilde{d}_{i} \frac{\eta_{i}}{\left(1+\zeta_{i}^{2}\right)\left(\zeta_{i}^{2}+\eta_{i}^{2}\right)}, \\
& D_{z}(r, z)=-\frac{2}{\pi \tilde{m}} \sum_{i=1}^{4} a_{6 i} \lambda_{i} \tilde{d}_{i}\left(\frac{\pi}{2}-\tan ^{-1} \zeta_{i}-\frac{\zeta_{i}}{\zeta_{i}^{2}+\eta_{i}^{2}}\right)+D^{*}, \\
& B_{r}(r, z)=-\frac{2 r}{\pi a \tilde{m}} \sum_{i=1}^{4} a_{7 i} \lambda_{i}^{2} \tilde{d}_{i} \frac{\eta_{i}}{\left(1+\zeta_{i}^{2}\right)\left(\zeta_{i}^{2}+\eta_{i}^{2}\right)}, \\
& B_{z}(r, z)=-\frac{2}{\pi \tilde{m}} \sum_{i=1}^{4} a_{7 i} \lambda_{i} \tilde{d}_{i}\left(\frac{\pi}{2}-\tan ^{-1} \zeta_{i}-\frac{\zeta_{i}}{\zeta_{i}^{2}+\eta_{i}^{2}}\right)+B^{*} .
\end{aligned}
$$

The following integrals are used

$$
\begin{aligned}
& \int_{0}^{\infty} \frac{d}{d \xi}\left(\frac{\sin \xi a}{\xi}\right) e^{-\xi \lambda_{i} z} J_{0}(r \xi) d \xi=-a n_{i}\left[1-\zeta_{i}\left(\frac{\pi}{2}-\tan ^{-1} \zeta_{i}\right)\right] \\
& \int_{0}^{\infty} \frac{d}{d \xi}\left(\frac{\sin \xi a}{\xi}\right) e^{-\xi \lambda_{i} z} J_{l}(r \xi) d \xi=-\frac{r}{2}\left(\frac{\pi}{2}-\tan ^{-1} \zeta_{i}-\frac{\zeta_{i}}{1+\zeta_{i}^{2}}\right), \\
& \int_{0}^{\infty} \xi \frac{d}{d \xi}\left(\frac{\sin \xi a}{\xi}\right) e^{-\xi \lambda_{i} z} J_{0}(r \xi) d \xi=-\frac{\pi}{2}+\tan ^{-1} \zeta_{i}+\frac{\zeta_{i}}{\zeta_{i}^{2}+\eta_{i}^{2}} \\
& \int_{0}^{\infty} \xi \frac{d}{d \xi}\left(\frac{\sin \xi a}{\xi}\right) e^{-\xi \lambda_{i} z} J_{l}(r \xi) d \xi=-\frac{r}{a} \frac{\eta_{i}}{\left(1+\zeta_{i}^{2}\right)\left(\zeta_{i}^{2}+\eta_{i}^{2}\right)},
\end{aligned}
$$

where

$$
\begin{aligned}
& \zeta_{i}\left(r, z, a, \lambda_{i}\right)=\frac{1}{\sqrt{2} a} \sqrt{\sqrt{\left(r^{2}+\lambda_{i}^{2} z^{2}-a^{2}\right)^{2}+4 \lambda_{i}^{2} z^{2} a^{2}}+\left(r^{2}+\lambda_{i}^{2} z^{2}-a^{2}\right)}, \\
& \eta_{i}\left(r, z, a, \lambda_{i}\right)=\frac{1}{\sqrt{2} a} \sqrt{\sqrt{\left(r^{2}+\lambda_{i}^{2} z^{2}-a^{2}\right)^{2}+4 \lambda_{i}^{2} z^{2} a^{2}}-\left(r^{2}+\lambda_{i}^{2} z^{2}-a^{2}\right)}
\end{aligned}
$$

and $\lambda_{i}$ are the roots of Eq.(2.7) with positive real parts.

\section{Nomenclature}

$$
a \text { - radius of the penny - shaped crack }
$$




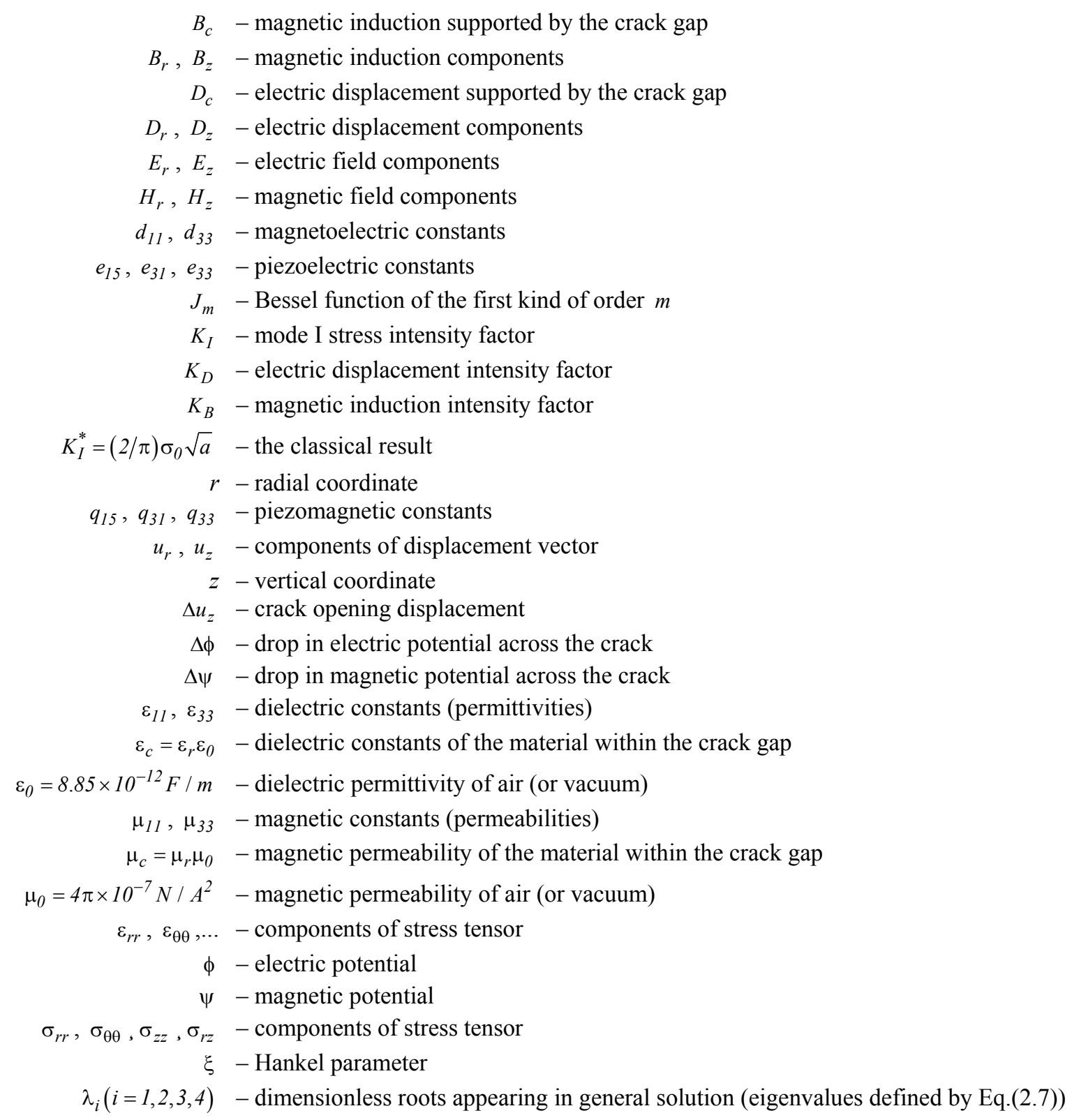

\section{References}

Chen W.Q., Lee K.Y. and Ding H.J. (2004): General solution for transversely isotropic magneto electro thermo elasticity and the potential theory method. - International Journal of Engineering Sciences, vol.42, pp.13611379.

Hao T.H. and Shen Z.Y. (1994): A new electric boundary condition of electric fracture mechanics and its application.Engineering Fracture Mechanics, vol.47, pp.793-802.

Niraula P.P. and Wang B L.( 2006): A magneto electro elastic material with a penny shaped crack subjected to temperature loading. - Acta Mech., vol.187, pp.151-168.

Rogowski B.(2007): The limit electrical permeable crack model in linear piezoelectroelasticity. - International Journal of Pressure, Vessel and Piping,vol.84, pp.572-581.

Rogowski B. (2011): The mode III crack emanating from an elliptical hole in the piezo - electro - magneto - elastic materials. - Archives of Applied Mechanics, vol.81, pp.1607-1620. 
Wang B.L. and Mai Y.W. (2007): Applicability of the crack - face electromagnetic boundary conditions for fracture of magnetoelectroelastic materials. - International Journal of Solids and Structures, vol.44, pp.387-398.

Zhao M.H., Yang F. and Liu T. (2006): Analysis of a penny - shaped crack in a magneto - electro - elastic medium. Philosophical Magazine, vol.86, pp.4397-4416.

Zhong G.C. and Li X.F. (2007): T-stress analysis for a Griffith crack in magnetoelectroelastic solid. - Archive of Applied Mechanics, vol.78, pp.117-125.

Zhong X.C. and Li. X.F. (2008): Fracture analysis of magnetoelectroelastic solid with a penny - shaped crack by considering the effects of the opening crack interior. - International Journal of Engineering Science, vol.46, pp.374 390.

Zhong X.C. (2009): Analysis of a dielectric crack in a magnetoelectroelastic layer. - International Journal of Solids and Structures, vol., pp.4221 - 4230.

Received: December 1, 2011

Revised: September 2, 2012 